\title{
Allowance treatment static designed couple and repeatable precision in assembly
}

\author{
Štefan Václav ${ }^{l}$, Jozef Jurko, PhD. ${ }^{2}$ and Šimon Lecký ${ }^{1, *}$ \\ ${ }^{1}$ Slovak University of Technology, Faculty of materials science and technology in Trnava, \\ Institute of production technologies, Slovakia \\ ${ }^{2}$ The Technical University of Košice Faculty of Manufacturing Technologies with a seat in Prešov, \\ Slovakia
}

\begin{abstract}
In practice, we deal with static designed couples frequently. Construction engineers use them so they can upscale rigidity of product. In paper are described principles how to change after effects of problematic assembly in often used feed couples which use to be statically designed. Important problem in assembly is repeatable precision, mainly in construction of assembly lines. By imprecision of robot which inserting shank into the hole and by imprecision of position of hole in carrier shank won't be successfully inserted. In article are shown reasons and security approach of repeatable precision in assembly operations executing.
\end{abstract}

\section{Introduction}

Nowadays it is unlikely that any producer or manufacturer will produce their products without any assembly process. The more usual case is that a producer will have several components manufactured by themselves or suppliers and then assembled together [1]. The product designer is normally limited only by a few critical dimensions and has much scope in the design of individual components and assemblies [2]. For example, challenging precision boning is the interdependency between alignment and boning [3]. There are many good and bad examples, in the works of DFA. The good ones are statically determinate, the bad ones are over determinate [4]. In practice, the cases of statically predetermined couples are often encountered. The designers like using them to enhance rigidity of the product. A typical example is a ball bearing, which should theoretically have the maximum of three balls, but it has more of them in order to increase their toughness.

Figure 1 illustrates a hydraulic press that is statically predetermined. When the support moves on two cylinders, the couple is clearly statically predetermined, as shown in the Grübler - Valentovič equation, according to which the number of degrees of freedom is minus two.

There are more conditions for trouble-free assembly regarding the fact that the couples are not parallel but concurrent or even skewed. In such circumstances, the couple must have

\footnotetext{
${ }^{*}$ Corresponding author : simon.lecky@stuba.sk
} 
gaps enabling smooth assembly even despite non-parallelism of the couple, which in turn partially decreases toughness.

It is often necessary to bond two elements (Figure 1) which apparently form a statically predetermined kinematic couple.

This concerns also a flanged bond, e.g. the bond of white and black flanges with four pins (Figure 2a). It is desirable to label both parts in order to enable assembly in any case (trouble-free assembly).

The above-mentioned suggests that there always should be pins inside the holes (Figure 2d). This can only be achieved by labelling the pins (Figure 2, pos. 2) and holes (Figure 2, pos. 1) separately, each from the same base. The same will be done with the trusses (Figure 2b). The reason for this method of labelling is due to the fact that manufacturing tolerances are not added up.

The depicted couples are very strongly statically predetermined structures, where the expected number of degrees of freedom equals one, in order to enable feed.

The formula in Figure 1c suggests that the number of degrees of freedom "is $=-10$ ", implying that the system is statically prearranged 9 times. The right labelling can assure trouble-free assembly.

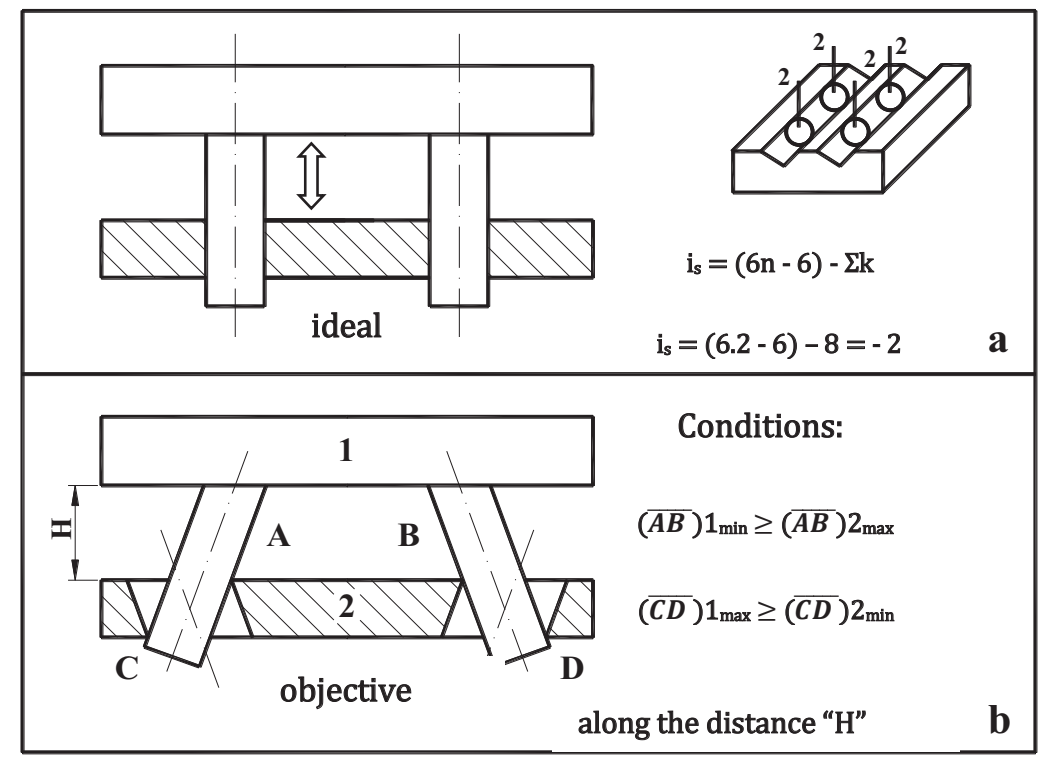

Fig. 1. Toleration treatment of statically redetermined movable couples.

This concerns also a flanged bond, e.g. the bond of white and black flanges with four pins (Figure 2a). It is desirable to label both parts in order to enable assembly in any case (trouble-free assembly).

The above-mentioned suggests that there always should be pins inside the holes (Figure 2d). This can only be achieved by labelling the pins (Figure 2, pos. 2) and holes (Figure 2, pos. 1) separately, each from the same base. The same will be done with the trusses (Figure $2 b)$. The reason for this method of labelling is due to the fact that manufacturing tolerances are not added up.

The depicted couples are very strongly statically prearranged structures, where the expected number of degrees of freedom equals one, in order to enable feed. 
The formula in Figure 2c suggests that the number of degrees of freedom " $\mathrm{i}_{\mathrm{s}}=-10$ ", implying that the system is statically prearranged 9 times. The right labelling can assure trouble-free assembly.

Such treatment enables smooth assembly of the components manufactured in large series.

Figure 3 shows a different method of mitigating the consequences of troublesome assembly of very frequent sliding couples which are generally statically prearranged. The couple depicted in Figure 3a is evidently statically prearranged; the consequences may be mitigated when first assembling the sliding workbench with rods and then connecting them to the base (Figure $3 b$ ).

Figure $3 \mathrm{c}$ is the best illustration of trouble-free assembly: there are two rotary-sliding couples (four-point) on one shaft, and a one-point couple on the other one (touches the left or the right side). Such a structure is statically prearranged but defined, which may assure completely trouble-free assembly.

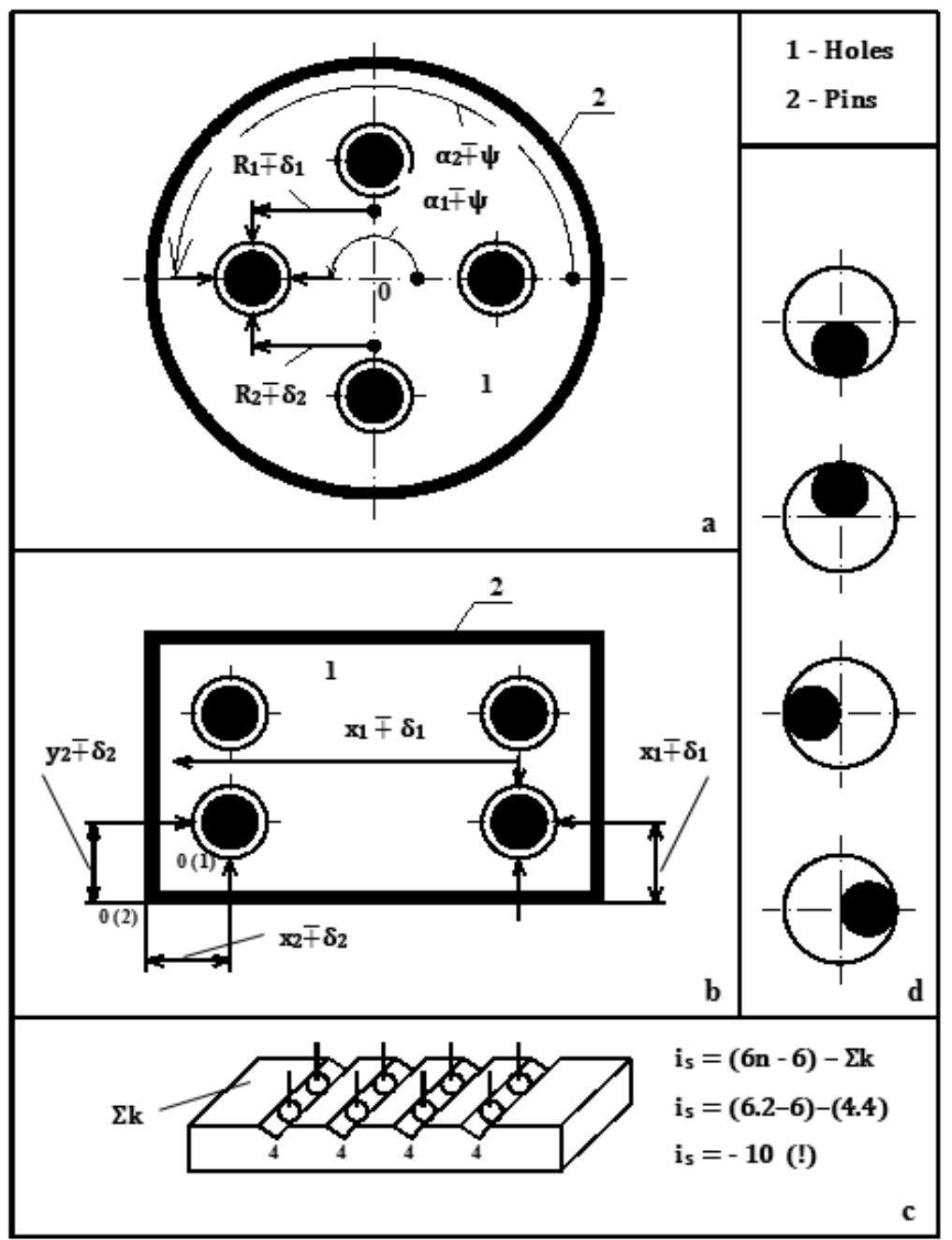

Fig. 2. Assembly ability of statically redetermined unmovable couples: a, b-dimension method, cnumber of degrees of freedom, $\mathrm{d}$ - assemblability check. 


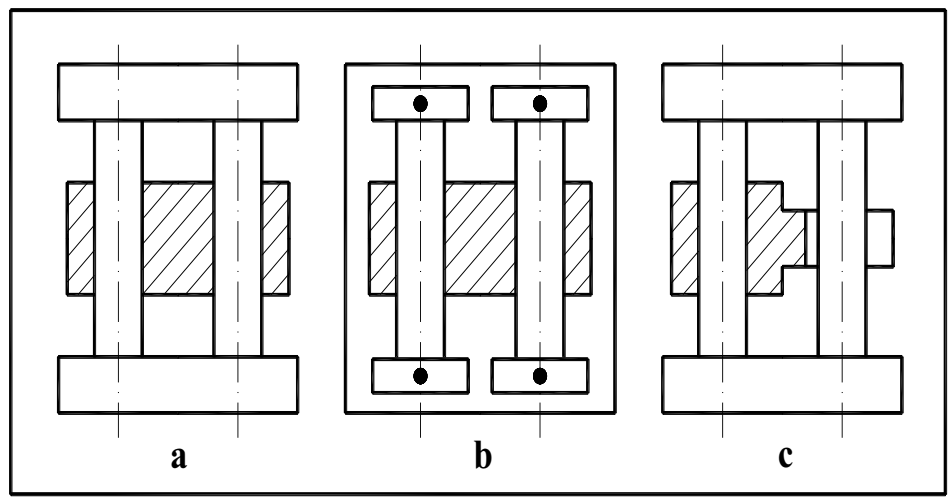

Fig. 3. Assembly ability of movable couples: $a$ - statically prearranged structure, $b$ - gradual assembly of statically prearranged structure, $\mathrm{c}$ - statically determined structure (trouble-free assembly).

If high accuracy in the product is not needed, so called extenders of gaps can be used. The principle is shown in Figure 4.

\section{Repeated precision of assembly}

A very important issue in assembly is repeated accuracy, especially when constructing assembly lines. Inaccuracy of the robot inserting the pin into the hole may cause the inaccuracy of the hole location in the gripper, which may prevent a successful insertion of the pin into the hole. This may be due to the reasons described below [4].

The first reason for the failure of assembly is that a robot collecting pins from the palette has a certain repetitive accuracy (uncertainty) of positioning, i.e. when inserting the pins, their spikes are not positioned to the same point (position), but make a circle of eccentricity "Ek" (Figure 4a).

Another reason for the failure of assembly is that the center of the hole is not in the same place with respect to the frame of the machine, but may be in a different place in each clamp, which is due to inaccuracy of the gripper and clamp, and sometimes also due to inaccurate contours of the part in the clamp (Figure 4a).

The issue poses a problem since the two causes may be combined (inaccuracies may increase by mutual summation) and it can be easily proved (Figure $4 \mathrm{~b}$ ) that to insert a pin by the radius $R_{k \max }$ into the hole, the radius of the hole must be:

$$
\mathrm{R}_{\mathrm{dmin}}=\mathrm{E}_{\mathrm{d}}+\mathrm{E}_{\mathrm{k}}+\mathrm{R}_{\mathrm{kmax}}[\mathrm{mm}]
$$

where:

$\mathrm{R}_{\mathrm{dmin}}-$ minimum radius of opening [mm],

$\mathrm{E}_{\mathrm{d}}$ - eccentricity of opening [mm],

$\mathrm{E}_{\mathrm{k}}$ - eccentricity of pin [mm],

$\mathrm{R}_{\mathrm{kmax}}-$ maximum radius of pin [mm].

From experience it is known that such assembly requires a disproportionately large gap between the pin and the hole. This can be eliminated by using the maximum chamfer of both components (Figure 4c) and then trying to insert the pin into the hole by utilizing flexibility.

Another option is a little more complicated: the pin is placed next to the hole and then oscillatory motions are performed until success is achieved, under some pressure, to capture the pin into the hole (Figure 4d). 


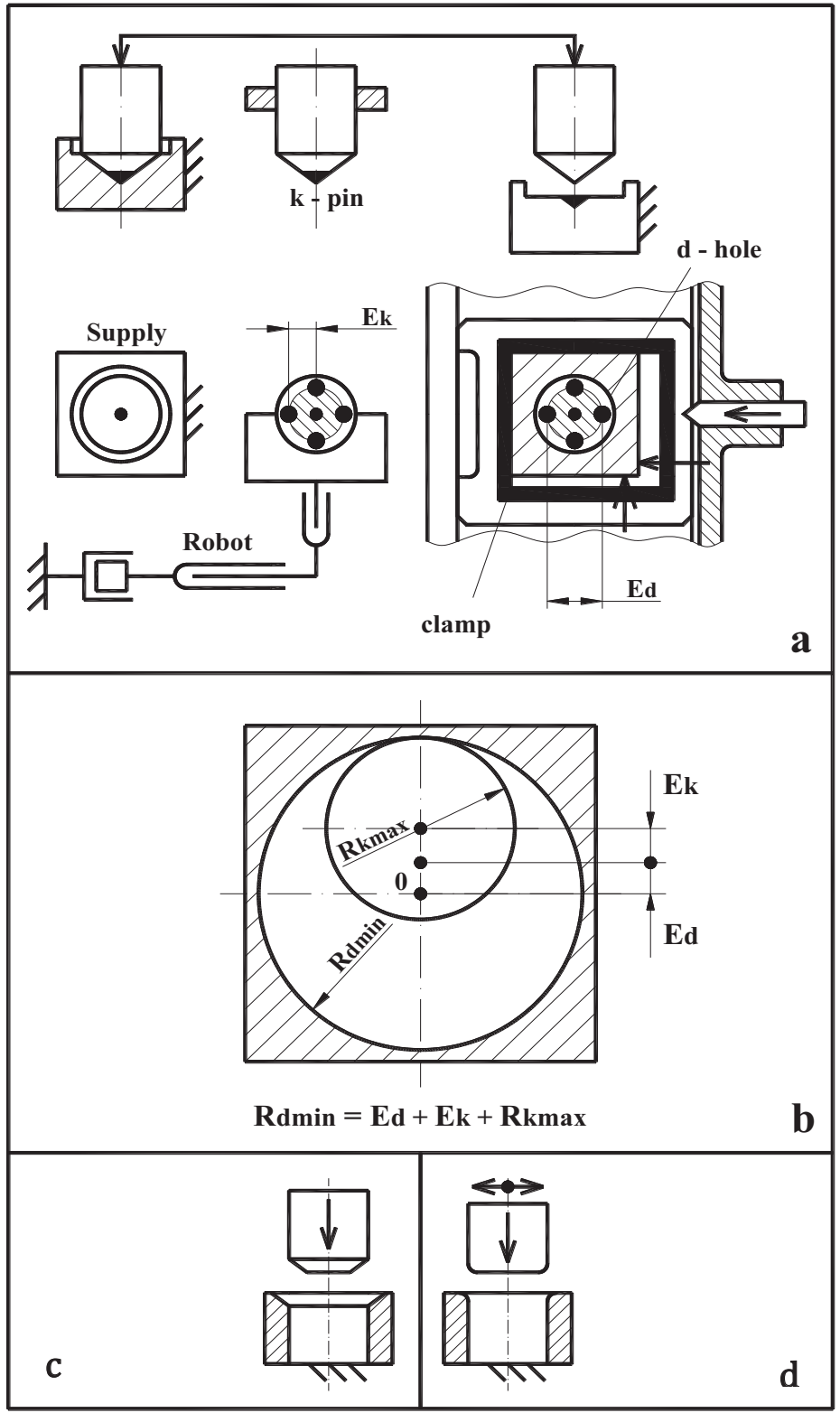

Fig. 4. Repeated accuracy of the robot and clamp increases the probability of the insertion of pin into hole, a - robot and clamp, $\mathrm{b}$ - conditions of insertion, $\mathrm{c}$ - chamfers improve probability of insertion, $\mathrm{d}$ - vibration improves probability of insertion.

\section{Conclusion}

The above-mentioned suggests that the shorter and of better quality the line, the lower the values of $E_{k}$ a $E_{d}$. As illustrated in Figure $4 b$, assembly will be trouble-free under the following condition:

$$
\mathrm{R}_{\mathrm{dmin}}=\mathrm{E}_{\mathrm{d}}+\mathrm{E}_{\mathrm{k}}+\mathrm{R}_{\mathrm{kmax}}[\mathrm{mm}]
$$


or for the diameters:

$$
\begin{gathered}
\frac{\emptyset \text { dmin }}{2}=\mathrm{E}_{\mathrm{d}}+\mathrm{E}_{\mathrm{k}}+\frac{\emptyset \text { max }}{2} \\
\varnothing \mathrm{d}_{\min }=2 \mathrm{E}_{\mathrm{d}}+2 \mathrm{E}_{\mathrm{k}}+\emptyset_{\mathrm{kmax}}[\mathrm{mm}]
\end{gathered}
$$

After supplying particular values, e.g. $\varnothing_{\mathrm{kmax}}=40.1 \mathrm{~mm}, \mathrm{E}_{\mathrm{k}}=0.2 \mathrm{~mm}, \mathrm{E}_{\mathrm{d}}=0.1 \mathrm{~mm}$ : $\emptyset_{\mathrm{dmin}}=0.2+0.4+40.1=40.7[\mathrm{~mm}]$

Such a large gap $(0.7 \mathrm{~mm})$ cannot be accepted; the diameter of the hole will be therefore reduced. From experience, it is known that the gaps between pin and hole will be disproportionately large in such assembly.

The gap can be eliminated by using the maximum chamfer of both parts (Figure 4c) and then applying flexibility to insert the pin into the hole.

Another option is a little more complicated: the pin is placed next to the hole and then oscillatory motions are performed until success is achieved, under some pressure, to capture the pin into the hole (Figure 4d).

The machine to carry out such operations must be fitted with artificial sight and feeling. Such systems are very complex.

This contribution brings new insights into the tolerance treatment of statically assigned pairs for trouble-free mounting.

Another benefit of the article is the discovery of causes and the way to ensure the required repeat accuracy in carrying out assembly operations.

This article has been developed as part of the project: VEGA 1/0477/14 Examining the influence of selected machining process characteristics using HI-machining technology on the resulting quality of machined surfaces and seamless assembly.

\section{References}

1. H. A. Crostack, R. Refflinghaus, J. Liangsiri, Defining Tolerances in Assembly Process with the Aid of Simulation. In: S. Ratchev (eds) Precision Assembly Technologies and Systems. IFIP Advances in Information and Communication Technology 315. Springer, Berlin, Heidelberg (2010)

2. C.S. Syan, K. G. Swift, Design for manufacture. In: C.S. Syan, U. Menon (eds) Concurrent Engineering. Springer, Dordrecht (1994)

3. T. Müller et al., Robust Adhesive Precision Bonding in Automated Assembly Cells. In: S. Ratchev (eds) Precision Assembly Technologies and Systems. IFIP Advances in Information and Communication Technology 435. Springer, Berlin, Heidelberg (2014)

4. G. Boothroyd and P. Dewhurst, Product Design for Assembly, Wakenfield. USA (1987)

5. E. Valentovič. Assembly Automation 20 (3) 233 (2000) 